\title{
Beyond halothane: an update on pediatric anesthesia pharmacology
}

$\mathrm{P}$ HARMACOLOGIC agents when introduced into clinical practice are routinely first investigated in the adult population. Investigation and a wide acceptance into practice typically occurs much later in children and lastly or never in infants and neonates. Pediatric anesthesiologists are frequently required to use "orphan" drugs and develop techniques for general anesthesia before appropriate investigation of drug pharmcokinetics, pharmacodynamics, or safety are available. A recent example of the potential problems of this situation would be the removal of rapacuronium due to the high incidence of bronchospasm, shortly after clinical introduction. We as practitioners may choose to continue to use traditional agents such as halothane or succinylcholine because of readily available research, historical evidence of safety and personal clinical experience. However, this results in many anesthesiologists having widely different practices for the routine anesthesia of their adult patients compared to their pediatric ones. In addition, the part-time pediatric anesthesiologist is often called upon to perform pediatric anesthesia in an urgent or emergency setting where there are also significant patient factors to consider.

The higher morbidity in our pediatric patients is well recognized. ${ }^{1}$ Analysis of morbidity data has shown an association in the occasional use of halothane by non-subspecialty anesthesiologists and the failure to recognize over dosage. ${ }^{1}$ These potent cardiorespiratory depressive effects of halothane are usually undesirable and other choices for pediatric anesthesia are available. Other issues with routine halothane use include: lack of availability on the anesthetic machine in many adult hospitals; occupational exposure with liver toxicity; or excessive cost associated with an infrequently used agent. This refresher course article will look at pharmacologic information for the use of newer agents with a view to increasing the comfort of the occasional pediatric anesthesiologist. Then we can answer whether it is time for all anesthesiologists to move beyond halothane.

\section{Inhalational agents}

Excellent reviews of the pharmacology of inhalational anesthetics in pediatrics are available..$^{2,3} \mathrm{~A}$ review of the literature reminds us that the minimum alveolar concentration (MAC) of inhalational agents is different in infants and children who require higher concentrations of all potent vapours. This elevation in MAC declines in the neonate or premature patient. See the Table for typical MAC values for the commonly used agents.

TABLE

\begin{tabular}{lllll}
\hline & Blood/gas solubility & Neonate & Infant & Older child \\
\hline Halothane & 2.4 & .87 & 1.2 & .75 \\
Isoflurane & 1.4 & 1.6 & 1.9 & 1.3 \\
Sevoflurane & .66 & 3.3 & 3.3 & 2.5 \\
Desflurane & .42 & 9.2 & 9.9 & 8 \\
\hline
\end{tabular}

Solubility and minimum alveolar concentration $v$ s agent in various ages. $^{3}$

Only halothane and sevoflurane are widely regarded to be useful for inhalational induction. The rapid induction with halothane depends on the use of "over pressure" to achieve rapid unconsciousness. Due to the lower solubility of sevoflurane, the same rapid loss of consciousness may be achieved without turning the vapourizer to a potentially toxic setting. This factor combined with the reduced negative inotropic effects of sevoflurane should make sevoflurane safer in the hands of non-pediatric anesthesiologists. ${ }^{4}$ Dysrrythmias with hypoventilation and resultant hypercarbia are also more common with halothane than when ether type anesthetics are used. ${ }^{3}$ While all anesthetic vapours can cause respiratory depression, there are differences. Sevoflurane produces less central excitation than halothane on the respiratory centre with typical respiratory rates at 30 rather than 40 with 1 to 1.2 MAC. $^{5}$ Also, thoracoabdominal synchrony is better preserved. ${ }^{5}$ Anesthesiologists in North America were fortunate to 
have access to publications describing a large clinical experience with the use of sevoflurane in Japan before its clinical introduction here suggesting that sevoflurane was a safe agent.

Fewer published studies are available investigating the pediatric use of desflurane likely secondary to the potent airway stimulating effects, and the high incidence of laryngospasm when employed as an inhalational induction agent. ${ }^{3}$ It appears to be an excellent agent for the maintenance of anesthesia in infants and children; especially in cases of long duration. ${ }^{6}$ Low flows are necessary for cost reduction.

Supplementing both sevoflurane and desflurane in pediatrics with nitrous oxide does not give additive MAC reduction and is likely less useful than with the more soluble vapours. ${ }^{7}$ This allows the avoidance of nitrous oxide with reduction of pollution and diminished nausea and vomiting. Emergence can still be very rapid.

The major drawback associated with sevoflurane and desflurane is emergence delirium. Usually rapid emergence with these low solubility agents is not a problem. However, with inadequate pain control the incidence is as high as $40 \%$ of toddlers. Anesthesiologists have used regional techniques ${ }^{8}$ ensuring pain control with empiric narcotics or the addition of agents such as clonidine with reduction but not elimination of emergence delirium, the incidence still remaining at about $10 \%$ which is similar to halothane. ${ }^{8}$ Further research is required as these patients with delirium are difficult to control and are not fit for discharge from the recovery area, thus negating any possible benefit that rapid emergence may have on length of stay in recovery areas.

\section{Intravenous anesthetics}

Pediatric patients challenge the anesthesiologist by the changing pharmacokinetic and pharmacodynamic responses to iv agents. The rapidly changing physiology of the infant warrants careful consideration when using "fixed" doses of $i v$ agents and pathologic states interact further to make the variability even more. ${ }^{9}$

Propofol, while hardly new, can be used in shorter cases to speed recovery when compared to thiopental. This is due to both faster clearance and redistribution. Infants and children need significantly more of the drug because of this higher clearance. ${ }^{10}$ Pediatric patients also require higher plasma concentrations compared to older subjects. ${ }^{11}$ Propofol is more efficacious at depressing pharyngeal and laryngeal reflexes than pentothal and is preferred for intubation, insertion of laryngeal masks or for bronchoscopy. ${ }^{12}$ The admixture of propofol and thiopental in equal milligrams can reduce the pain on injection for a more pleasant induction. ${ }^{13}$
Ketamine is, in the author's opinion, underutilized. It is becoming more popular for non-anesthesiologists providing sedation and anesthesia for procedural pain or diagnostic testing. ${ }^{14}$ It too is cleared more rapidly in children, but clearance is reduced in the neonate with immature microsomal activity of the liver. ${ }^{9}$ It has intense analgesic effects. Single agent ketamine use is associated with the preservation of pharyngeal and laryngeal reflexes making it an excellent agent where airway intervention is not necessary. It is also useful in sick premature infants for maintenance of a higher mean arterial pressure than with isoflurane or fentanyl. ${ }^{9}$

Opioids are a vital part of pediatric anesthesia both for co-induction and adjuvant anesthesia, and for postprocedure sedation and analgesia. The newest agent remifentanil is a drug whose effects are rapidly terminated by metabolism of an ester linkage. This seems to be independent of age or pathology, as this agent can be employed even in sick neonates. Remifentanil's ultra-short context sensitive half-time is still only minutes. ${ }^{15}$ A reduction in the incidence of postoperative apnea was noted in term infants when remifentanil and nitrous oxide was compared to halothane for pyloromyotomies. ${ }^{16}$ Whether this would also be true if the volatile agent studied was sevoflurane has not been investigated. Another opioid, sufentanil, has a shorter context sensitive half-time than alfentanil or fentanyl. ${ }^{17}$ This is a better choice for longer duration cases where significant postoperative pain is expected. The association of remifentanil with acute tolerance is a concern, which can best be avoided by the use of more slowly metabolized narcotics like sufentanil. ${ }^{18}$

Total intravenous anesthesia (TIVA) for children is improving with the addition of remifentanil. The pharmacokinetics in children are being worked out such that computer targeted infusions for certain serum levels can be employed to prevent prolonged awakening. ${ }^{11}$ With the addition of a context insensitive drug like remifentanil this will make the variability less, but the challenge is still to program pumps to set a ratio between propofol or remifentanil. Costs of TIVA in small children are low; and its practice is environmentally friendly. Those working around the airway, such as with an otorhinolaryngologist performing bronchoscopy, appreciate conditions where minimal volatile agent or nitrous oxide are present. Five-fold increases above levels suggested by government regulations for safety are found during bronchoscopy with halothane anesthesia. ${ }^{19}$ A typical combination used in our institution for spontaneous ventilation during broncoscopy is to use a single syringe with propofol and remifentanil in a ratio of 10 
mg of propofol with $2.5 \mu \mathrm{g}$ of remifentanil. By titrating the dose of propofol from 200 to 400 $\mu \mathrm{g} \cdot \mathrm{kg}^{-1} \cdot \mathrm{min}^{-1}$ the remifentanil will be running at 0.05 to $0.1 \mu \mathrm{g} \cdot \mathrm{kg}^{-1} \cdot \mathrm{min}^{-1}$. This usually provides for a rapid induction and good conditions for bronchoscopy. More studies comparing TIVA and the newer inhalational agents will be forthcoming to help guide further practice.

\section{Muscle relaxation}

With the addition of more short-acting anesthetic agents, we now have an ability to give better quality anesthesia and do not have to depend on muscle relaxants to "cover" for anesthesia. Endotracheal intubation is greatly facilitated by either the use of sevoflurane or propofol and remifentanil in combination, and we can avoid the routine use of muscle relaxants. ${ }^{20}$ When we do need to use relaxants short-acting agents with predictable metabolism such as cisatracurium would be a good choice. Rocuronium seems the best agent for rapid sequence induction. Even the emergency use of succinylcholine for laryngospasm can be reduced by the ability to give repeated doses of propofol. Rapacuronium was not approved for use in Canada because of severe bronchospasm in many children.

\section{Local anesthetics}

Local anesthetics depend on maturation of cytochrome metabolism, which is immature in the neonate. Ropivacaine is clinically available and has advantages, which include less motor block and likely a wider therapeutic index in neonates. Tetracaine $4 \%$ gel has a faster onset with less vasoconstriction than a lidocaine/prilocaine formulation. ${ }^{21}$

\section{Conclusion}

Newer drugs and the study of their pharmacology in pediatric anesthesia are helping us to provide excellent care. We are able to practice both anesthesia and reanimation while wasting little of the valuable facility resources. Sevoflurane has challenged halothane for safer inhalational inductions, while being faster with onset and recovery. Intravenous anesthesia also has many attributes in pediatrics. With careful planning for analgesia, and the appropriate use of adjuvants a controlled emergence from anesthesia can be obtained. This should remove the reluctance of many to move beyond halothane.

\section{References}

1 Keenan RL, Shapiro JH, Kane FR, Simpson PM. Bradycardia during anesthesia in infants. An epidemiologic study. Anesthesiology 1994; 80: 976-82.
2 Cook RJ, Davis PJ, Lerman J. Pharmacology of pediatric anesthesia In: Motoyama E, Davis PJ (Eds.). Smith's Anesthesia for Infants and Children, 6th ed. St.Louis: Mosby; 1996: 159-209.

3 Lerman J. Inhalational agents. In: Bissonnette B, Dalens BJ (Eds.). Pediatric Anesthesia Principles and Practice. New York: McGraw-Hill; 2002: 215-36.

4 Friesen RH, Wurl JL, Charlton GA. Haemodynamic depression by halothane is age-related in paediatric patients. Paediatr Anaesth 2000; 10: 267-72.

5 Brown K, Aun C, Stocks J, Jackson E, Mackersie A, Hatch $D$. A comparison of the respiratory effects of sevoflurane and halothane in infants and young children. Anesthesiology 1998; 89: 86-92.

6 Wolf AR, Lawson RA, Dryden CM, Davies FW. Recovery after desflurane anaesthesia in the infant: comparison with isoflurane. Br J Anaesth 1996; 76: 362-4.

7 Zwass MS, Fisher DM, Welborn LG, et al. Induction and maintenance characteristics of anesthesia with desflurane and nitrous oxide in infants and children. Anesthesiology 1992; 76: 373-8.

8 Aono J, Ueda W, Mamiya K, Takimoto E, Manabe M. Greater incidence of delirium during recovery from sevoflurane anesthesia in preschool boys. Anesthesiology 1997; 87: 1298-300.

9 Brooker PD. Intravenous anesthetics. In: Bissonnette B, Dalens BJ (Eds.). Pediatric Anesthesia Principles and Practice. New York: McGraw-Hill; 2002: 237-58.

10 Everett $L L$. Newer drugs in pediatric anesthesia. Semin Pediatr Surg 1999; 8: 6-12.

11 Hammer GB, Litalien C, Wellis V, Drover DR. Determination of the median effective concentration (EC50) of propofol during oesophagogastroduodenoscopy in children. Paediatr Anaesth 2001; 11 : 549-53.

12 Reyle-Habn M, Niggemann B, Max M, Streich R, Rossaint $R$. Remifentanil and propofol for sedation in children and young adolescents undergoing diagnostic flexible bronchoscopy. Paediatr Anaesth 2000; 10: 59-63.

13 Pollard RC, Makky S, McFadzean J, Ainsworth L, Goobie SM, Montgomery CJ. An admixture of 3 $\mathrm{mg} \cdot \mathrm{kg}(-1)$ of propofol and $3 \mathrm{mg} \cdot \mathrm{kg}(-1)$ of thiopentone reduces pain on injection in pediatric anesthesia. Can J Anesth 2002; 49: 1064-9.

14 Mason KP, Michna E, DiNardo JA, et al. Evolution of a protocol for ketamine-induced sedation as an alternative to general anesthesia for interventional radiologic procedures in pediatric patients. Radiology 2002; 225: 457-65.

15 Ross AK, Davis PJ, Dear Gd GL, et al. Pharmacokinetics of remifentanil in anesthetized pedi- 
atric patients undergoing elective surgery or diagnostic procedures. Anesth Analg 2001; 93: 1393-401.

16 Galinkin JL, Davis PJ, McGowan FX, et al. A randomized multicenter study of remifentanil compared with halothane in neonates and infants undergoing pyloromyotomy. II. Perioperative breathing patterns in neonates and infants with pyloric stenosis. Anesth Analg 2001; 93: 1387-92.

17 Hughes MA, Glass PS, Jacobs JR. Context-sensitive halftime in multicompartment pharmacokinetic models for intravenous anesthetic drugs. Anesthesiology 1992; 76: 334-41.

18 Guignard B, Bossard AE, Coste C, et al. Acute opioid tolerance: intraoperative remifentanil increases postoperative pain and morphine requirement. Anesthesiology 2000; 93: 409-17.

19 Hoerauf K, Kessler P, Alemdag $\Upsilon$, Wiesner G, Janhson $G$, Taeger $K$. Room air contamination with halothane during pediatric bronchoscopy (German). Zentralbl Hyg Umweltmed 1997; 199: 551-7.

20 Robinson DN, O'Brien K, Kumar R, Morton NS.

Tracheal intubation without neuromuscular blockade in children: a comparison of propofol combined either with alfentanil or remifentanil. Paediatr Anaesth 1998; 8: 467-71.

21 Speirs AF, Taylor KH, Joanes DN, Girdler NM. A randomised, double-blind, placebo-controlled, comparative study of topical skin analgesics and the anxiety and discomfort associated with venous cannulation. $\mathrm{Br}$ Dent J 2001; 190: 444-9. 